4 Gosselin LE, Brice G, Carlson B, et al. Changes in satellite cell mitotic activity during acute period of unilateral diaphragm denervation. J Appl Physiol 1994; 77: 1128-1134.

5 Welvaart WN, Paul MA, van Hees HW, et al. Diaphragm muscle fiber function and structure in humans with hemidiaphragm paralysis. Am J Physiol Lung Cell Mol Physiol 2011; 301: L228-L235.

6 Gayan-Ramirez G. Ventilator-induced diaphragm dysfunction: time for (contr)action! Eur Respir J 2013; 42: 12-15.

\title{
Asthma and risk of pulmonary thromboembolism
}

To the Editor:

We read with interest the article by CHUnG et al. [1] about the risk of pulmonary thromboembolism in asthmatic patients. This nationwide population cohort study suggests that the risk of developing pulmonary thromboembolism significantly is increased in asthmatic patients compared to those of the general population, with a multivariable-adjusted hazard ratio of 3.24 (95\% CI 1.74-6.01). The authors considered that as concentrations of thrombin were elevated in the sputum and bronchoalveolar lavage of asthmatic patients, and as local coagulation activation existed in asthma, it is possible that the results of this study may, in part, be explained through this mechanism. However, there are other plausible mechanisms that might explain the risk.

In asthmatic patients, plasma oxidant-antioxidant status was abnormal, with increased plasma malondialdehyde and decreased plasma ascorbic acid, which support the emerging concept of free-radical injury in asthma [2]. The pathogenesis of venous thromboembolism is also linked to oxidative stress [3]. Therefore, the involvement of oxidative stress may potentiate the increased risk of pulmonary thromboembolism in asthmatic patients.

Moreover, as the study by MAJOOR et al. [4] suggested, on one hand, that inactivity of severe asthmatic patients might be a potential trigger for venous thromboembolic events, but on the other hand, asthmatic patients, especially severe cases, continuously use high doses of glucocorticoids, receive bursts of systemic glucocorticoid during exacerbations and often need chronic oral glucocorticoid treatment for control of their asthma. Recent studies suggested that use of glucocorticoids may be at an increased risk of venous thromboembolism [5] and pulmonary embolism [6]. Just as CHUNG et al. [1] recognised when discussing the limitations of their study, glucocorticoid use information was lacking in the multivariable Cox proportional-hazards regression analysis.

Notably, MAJOor et al. [4] found that the risk of pulmonary embolism was increased in severe asthma only, not in mild-to-moderate asthma.

-

@ERSpublications

Asthma and risk of pulmonary thromboembolism: more epidemiological studies are required http://ow.ly/qy3wh

Wan-Jie Gu and Jing-Chen Liu

Dept of Anesthesiology, the First Affiliated Hospital, Guangxi Medical University, Nanning, Guangxi, China.

Correspondence: J-C. Liu, Dept of Anesthesiology, the First Affiliated Hospital, Guangxi Medical University, 22 Shuangyong Road, Nanning 530021, Guangxi, China. E-mail: jingchenliu1964@sina.cn

Received: Sept 082013 | Accepted after revision: Sept 292013

Conflict of interest: None declared.

\section{References}

Chung WS, Lin CL, Ho FM, et al. Asthma increases pulmonary thromboembolism risk: a nationwide population cohort study. Eur Respir J 2014; 43: 801-807.

2 Ruprai RK. Plasma oxidant-antioxidants status in asthma and its correlation with pulmonary function tests. Indian J Physiol Pharmacol 2011; 55: 281-287.

3 Martinez M, Cuker A, Mills A, et al. Nitrated fibrinogen is a biomarker of oxidative stress in venous thromboembolism. Free Radic Biol Med 2012; 53: 230-236. 
4 Majoor CJ, Kamphuisen PW, Zwinderman AH, et al. Risk of deep vein thrombosis and pulmonary embolism in asthma. Eur Respir J 2013; 42: 655-661.

5 Johannesdottir SA, Horváth-Puhó E, Dekkers OM, et al. Use of glucocorticoids and risk of venous thromboembolism: a nationwide population-based case-control study. JAMA Intern Med 2013; 173: 743-752.

6 Stuijver DJ, Majoor CJ, van Zaane B, et al. Use of oral glucocorticoids and the risk of pulmonary embolism: a population-based case-control study. Chest 2013; 143: 1337-1342.

\title{
TLNO/TLCO ratio is not the end of the road
}

\author{
To the Editor:
}

As our team analysed in detail the physiological meaning of the transfer factor of the lung for nitric oxide (TLNO)/transfer factor of the lung for carbon monoxide (TLCO) ratio [1], it seems interesting to extend the analysis to patients. This has been done elegantly by HugHes and VAN DER LEE [2]. This ratio, which has the advantage of simplicity, nevertheless has some disadvantages. 1) In a given cohort of homogenous patients, values are widely scattered and a given value cannot, therefore, be used as a strong predictor of a disease. For example, pulmonary hypertension patients have, as a mean, higher TLNO/TLCO than healthy controls; however, a normal value does not exclude the disease. 2) The interpretation of an alteration in this ratio always makes allusions to capillary lung volume $\left(V_{\mathrm{c}}\right)$ and membrane conductance for carbon monoxide ( $\mathrm{m} \mathrm{mCO}$ ) variables, which stand behind this ratio. So why don't we used these variables directly? The answer is well known: we do not know the right values of the carbon monoxide and nitric oxide conductance of haemoglobin (specific conductance $(\theta)$ ), which are necessary to the calculation of $V_{\mathrm{c}}$ and $D \mathrm{~m}$. However, although we thought in 1987 that the conductance for nitric oxide could be taken as infinite [3], we have since changed our minds, as, at that time, we used the recommended carbon monoxide specific conductance of ROUGHTON and FORSTER [4], which was incorrect, as explained later by FORSTER [5]. The work of BORLAND et al. [6] added experimental arguments leading us to consider that the conductance of nitric oxide has a finite value.

What are the right values for $\theta \mathrm{NO}$ and $\theta \mathrm{CO}$. Following the in vitro work of CARLSEN and COMrOE [7], $\theta$ NO is $4.5 \mathrm{mmHg} \cdot \mathrm{min}^{-1}$ and the FORSTER [5] recommended value for $\theta \mathrm{CO}$ in normoxia is $0.58 \mathrm{mmHg} \cdot \mathrm{min}^{-1}$, the ratio of these conductances $(\theta \mathrm{NO} / \theta \mathrm{CO})$ would be 7.7 . It can be demonstrated using the ROUGHTON and Forster [4] equation for the two gases that the TLNO/TLCO ratio cannot be greater than $\theta \mathrm{NO} / \theta \mathrm{CO}$, i.e. 7.7 [8]. As the upper normal value of TLNO/TLCO reaches approximately 5.5-6.0 in most reports [2, 9], a $\theta$ NO value $<4.5$ or a $\theta \mathrm{CO}$ value $>0.58$ are unlikely as they would lead to a decrease in $\theta \mathrm{NO} / \theta \mathrm{CO}$ to $<7.7$. It could be suggested that both conductances might be lower than these in vitro values; however, it seems highly unlikely that both in vitro values were overestimated, and no published $\theta \mathrm{CO}$ value is $<0.58 \mathrm{mmHg} \cdot \mathrm{min}^{-1}$ in normoxia. Interestingly, using the aforementioned $\theta \mathrm{NO} / \theta \mathrm{CO}$ leads to increased $\mathrm{Dm}[8]$, reaching the morphometric value of WeIBEL et al. [10]. This reasoning has sharp consequences for the interpretation of both TLCO and TLNO as, if the $\theta \mathrm{NO} / \theta \mathrm{CO}$ value of 7.7 is confirmed, it would lead us to consider that TLCO is mainly dependent on $V$ c, as TLNO would be equally dependent on $D \mathrm{~m}$ and $V_{\mathrm{c}}$. Thus, TLCO would appear to be a vascular marker and TLNO would be the only marker sensitive to membrane alterations.

TLNO/TLCO ratio appears to be a step in our knowledge of diffusion, not the end: the road is still open.

@ERSpublications

The practical use of the TLNO/TLCO ratio in a clinical setting remains uncertain http://ow.ly/tRTAs

Jean-Benoit Martinot ${ }^{1}$ and Hervé Jean-Pierre Guénard ${ }^{2}$

${ }^{1}$ Dept of Pneumology, St Elisabeth Hospital, Namur, Belgium. ${ }^{2}$ Dept of Physiology, University Bordeaux 2, Bordeaux, France.

Correspondence: J-B. Martinot, St Elisabeth Hospital, 15 Place Louise Godin, 5000 Namur, Belgium. E-mail: martinot.j@scarlet.be

Received: Jan 162014 | Accepted: Jan 232014

Conflict of interest: None declared. 